\title{
Derivatization with Girard Reagent T Combined with LC-MS/MS for the Sensitive Detection of 5-Formyl-2'-deoxyuridine in Cellular DNA
}

\author{
Haizheng Hong ${ }^{1}$ and Yinsheng Wang ${ }^{1,2,{ }^{*}}$ \\ 1 Environmental Toxicology Graduate Program, University of California at Riverside \\ 2Department of Chemistry, University of California at Riverside
}

\begin{abstract}
Nucleoside 5-formyl-2'-deoxyuridine (FodU) is a major thymidine lesion generated by reactive oxygen species. In-vitro and in-vivo replication studies revealed that FodU can be mutagenic. A reliable and sensitive quantification method is, therefore, important for assessing the biological implications of this lesion. However, the detection limit of FodU by LC-MS/MS was relatively poor compared with those of other oxidative DNA base damages. In this paper we described a new approach for the highly sensitive detection of FodU. We derivatized FodU with Girard reagent $\mathrm{T}$ to form a hydrazone conjugate harboring a precharged quaternary ammonium moiety, which enabled the facile detection of the resulting conjugate by positive-ion ESI-MS. We also showed that the combination of derivatization with LC-MS/MS on a linear-ion-trap mass spectrometer could allow for the quantification of FodU at a detection limit of 3-4 fmol, which is $\sim 20$ fold better than that for the direct analysis of the underivatized compound. By using isotope-labeled FodU as the internal standard and this derivatization method, we further quantified, by using LC-MS/MS, the yield of FodU formed in cellular DNA.
\end{abstract}

\section{Introduction}

Reactive oxygen species (ROS) can be generated during normal aerobic metabolism or through exogenous pathways and excess generation of ROS in vivo results in damage to biological molecules including DNA ${ }^{1-3}$. Nucleoside 5-formyl-2'-deoxyuridine (FodU) is a major thymidine lesion generated by $\operatorname{ROS}^{4}, 5$, and the yields for the formation of FodU are similar as those of 8-oxo-7,8-dihydro-2'-deoxyguanosine (8-oxodG) ${ }^{6}$. In addition, in-vitro and invivo replication studies showed that FodU is mutagenic $4-6$.

Liquid chromatography-tandem mass spectrometry (LC-MS/MS) has been frequently employed for measuring oxidative DNA base lesions ${ }^{7,8}$. However, the detection limit of FodU was relatively poor compared with those of other oxidative DNA base damages, e.g., 8oxodG $^{7}$. A more sensitive method is, therefore, required to detect and quantify low-abundance FodU formed in cultured cells or primary tissues.

Girard reagents $\mathrm{T}$ (GirT, Figure 1) and $\mathrm{P}$ (GirP), which harbor a precharged quaternary ammonium and a pyridinium moiety, respectively, can react with ketones and aldehydes to yield hydrazone derivatives. These reagents have been used to derivatize steroids, peptides and oligosaccharides and the resulting conjugates can have improved sensitivities when analyzed

\footnotetext{
* To whom correspondence should be addressed: Department of Chemistry-027, University of California, Riverside, CA 92521-0403. Telephone: (951) 827-2700. Fax: (951) 827-4713. E-mail: yinsheng.wang@ucr.edu.
} 
by MS with electrospray ionization (ESI) or matrix-assisted laser desorption/ionization (MALDI) in the positive-ion mode ${ }^{9-12}$. However, the application of the derivatization chemistry together with LC-MS/MS for the quantitative assessment of ketone/aldehydebearing compounds has only begun to be explored ${ }^{13}$. In addition, stable isotope-labeled standards were not employed in that study 13 .

Herein, we showed that GirT could couple efficiently with FodU to form GirT hydrazone and LC-MS ${ }^{3}$ analysis, on an ESI-linear-ion-trap mass spectrometer, of the resulting conjugate allowed for the detection of FodU at low fmol level. With the availability of isotope-labeled FodU as an internal standard and with the derivatization method, we were further able to quantify the yields for the formation of FodU in DNA isolated from $\gamma$-irradiated human Hela S3 cells.

\section{Experimental Section}

Girard's reagent T (GirT) was obtained from Sigma-Aldrich (St. Louis, MO). Nuclease P1 and alkaline phosphatase were purchased from MP Biomedicals (Aurora, $\mathrm{OH}$ ) and Sigma-Aldrich (St. Louis, MO), respectively. Snake venom phosphodiesterase and calf spleen phosphodiesterase were obtained from US Biological (Swampscott, MA). Other chemicals were purchased from Sigma-Aldrich (St. Louis, MO). Hela-S3 cells were from the National Cell Culture Center (Minneapolis, MN). [1,3- ${ }^{15} \mathrm{~N}_{2}, 2$ '-D]-5-formyl-2'-deoxyuridine was prepared according to previously described procedures 8 .

\section{Optimization of reaction conditions for the derivatization of FodU (Figure 1)}

To optimize the reaction conditions, we first varied the reagent-to-lesion molar ratios by mixing aliquots of $1 \mathrm{mM}$ FodU with GirT at different molar ratios (2:1, 10:1, 30:1, 100:1, 300:1, 1000:1 and 2000:1, GirT/FodU), where 10\% acetic acid was always present in the reaction mixture. The mixtures were left at room temperature in the dark for $12 \mathrm{~h}$, and the reactions were stopped by freezing at $-80^{\circ} \mathrm{C}$.

The derivatization time was also optimized. In this respect, GirT was mixed with FodU at a molar ratio of 10:1 at room temperature in the presence of $10 \%$ acetic acid. Aliquots were removed from the reaction mixture at different time points (10 min, 1, 2, 4, 12 and $24 \mathrm{~h}$ ), and the reactions were terminated again by freezing at $-80^{\circ} \mathrm{C}$.

To examine how solution $\mathrm{pH}$ affects the derivatization reaction, GirT was mixed with FodU at a molar ratio of 10:1 or 100:1 in phosphate buffer $(50 \mathrm{mM}, \mathrm{pH} 7.0)$ or in a $10 \%$ (by volume) aqueous solution of acetic acid. The reactions were carried out at room temperature for $12 \mathrm{~h}$ and stopped by freezing at $-80^{\circ} \mathrm{C}$. The extent of conversion of FodU to the hydrazone conjugate was evaluated by HPLC analyses with conditions described below.

\section{Treatment of Hela-S3 cells with y-rays}

Hela-S3 cells, after received, were centrifuged to remove the culture medium and resuspended in phosphate-buffered saline (PBS). The cell suspension $\left(10^{7}\right.$ cells $\left./ \mathrm{mL}\right)$ was exposed to $\gamma$-rays delivered by a Mark I ${ }^{137}$ Cs Irradiator (JL Shepherd and Associates, San Fernando, CA), and the dose rate was $2.8 \mathrm{~Gy} / \mathrm{min}$. Immediately after the exposure, the cells were harvested by centrifugation and the nuclear DNA was isolated by phenol extraction.

\section{Enzymatic digestion and derivatization of DNA}

Five units of nuclease P1, 0.005 unit of calf spleen phosphodiesterase, and a $10-\mu \mathrm{L}$ solution containing $300 \mathrm{mM}$ sodium acetate (pH 5.0) and $10 \mathrm{mM}$ zinc acetate were added to $300 \mu \mathrm{g}$ of DNA sample and the digestion was carried out at $37^{\circ} \mathrm{C}$ for $6 \mathrm{~h}$. To the digestion mixture were 
then added 50 units of alkaline phosphatase, 0.05 unit of snake venom phosphodiesterase and $20 \mu \mathrm{L}$ of $0.5 \mathrm{M}$ Tris- $\mathrm{HCl}$ buffer ( $\mathrm{pH} 8.9$ ). The digestion was continued at $37^{\circ} \mathrm{C}$ for $6 \mathrm{~h}$, and the digestion mixture was passed through a YM-10 Centricon membrane (Millipore, Billerica, MA) to remove the enzymes. The amount of nucleosides in the mixture was quantified by UV absorption spectroscopy and to the mixture was then added isotopically labeled FodU.

In case that a trace amount of $\mathrm{dT}$ could be converted to FodU during the derivatization process, the samples were separated by HPLC (conditions described below) to remove most unmodified nucleosides. GirT, in 10000-fold excess of the internal standard, was then added to the FodUbearing fractions, to which acetic acid was also added to make its final concentration 10\% (by volume). The reaction was continued at room temperature for $12 \mathrm{~h}$ in the dark. The samples were then subjected to LC-MS/MS analysis.

HPLC

The off-line HPLC separation of the derivatization reaction mixtures was performed with a $4.6 \times 250 \mathrm{~mm}$ Polaris column ( $5 \mu \mathrm{m}$ in particle size, Varian Inc., Lake Forest, CA). A 45-min gradient of $0-6 \%$ acetonitrile in $10 \mathrm{mM}$ ammonium formate ( $\mathrm{pH} 6.3$ ) was employed and the flow rate was $0.80 \mathrm{~mL} / \mathrm{min}$.

The HPLC removal of unmodified nucleosides from the digestion mixture of cellular DNA was carried out by using a $4.6 \times 50 \mathrm{~mm}$ Luna reverse-phase $\mathrm{C} 18$ column $(5 \mu \mathrm{m}$ in particle size, Phenomenex ${ }^{\circledR}$, Torrance, CA). A gradient of 5-min 0-2\% acetonitrile followed by a 55-min $2-5 \%$ acetonitrile in $10 \mathrm{mM}$ ammonium formate ( $\mathrm{pH} 6.3$ ) was employed and the flow rate was $0.60 \mathrm{~mL} / \mathrm{min}$.

\section{LC-MS/MS analysis}

A $0.5 \times 150 \mathrm{~mm}$ Zorbax SB-C18 column $(5 \mu \mathrm{m}$ in particle size, Agilent Technologies, Palo Alto, CA) was used for the separation of the derivatized DNA hydrolysis samples, and the flow rate was $8.0 \mu \mathrm{L} / \mathrm{min}$, which was delivered by an Agilent 1100 capillary HPLC pump (Agilent Technologies). A 60-min gradient of 0-60\% acetonitrile in $20 \mathrm{mM}$ ammonium acetate was employed.

The effluent from the LC column was directed to an LTQ linear ion-trap mass spectrometer (Thermo Electron, San Jose, CA), which was set up for monitoring the fragmentation of the $[\mathrm{M}+\mathrm{Na}]^{+}$ion of the FodU or the $\mathrm{M}^{+}$ions of the GirT-conjugates of the unlabeled and labeled FodU.

\section{Results and Discussion Optimization of the derivatization reaction}

GirT is among a family of reagents originally developed to derivatize and solubilize steroids

11. They harbor a hydrazide moiety that can react with aldehydes and ketones to give hydrazone derivatives with a precharged quaternary ammonium moiety 9,14 , which can be readily detected by ESI- or MALDI-MS in the positive-ion mode. Thus, we expect to observe the conjugation reaction between the 5-formyl group of FodU and GirT (Figure 1). To test this, we treated FodU with GirT and separated the reaction mixture by HPLC. It turned out that GirT could indeed react readily with FodU to give the hydrazone conjugate [Figure 2a showed the HPLC trace for the separation of a reaction mixture where FodU has been treated with GirT at a molar ratio of $1: 2$ at room temperature for $12 \mathrm{~h}$ ]. The fraction eluting at $5.4 \mathrm{~min}$ was ascribed to some unknown impurity present in acetic acid, which had the maximum absorption at 205 $\mathrm{nm}$ and was also present in the HPLC trace while acetic acid was injected for analysis (Figure S1). The two small peaks at 16.9 and 17.5 min (Figure S1) might be degradation products of 
FodU and the quantities of the degradation products were much smaller than the amount of FodU used for the reaction.

The formation of the GirT hydrazone increases the mass of FodU by $133 \mathrm{Da}$, which gives $\mathrm{m} /$ $z$ of 370 for the $\mathrm{M}^{+}$ion of the GirT hydrazone conjugate of FodU. Collisional activation of the ion of $\mathrm{m} / \mathrm{z} 370$ leads to the formation of a single fragment ion of $\mathrm{m} / \mathrm{z} 254$, which can be attributed to the elimination of a 2-deoxyribose moiety (Figure 2b). Further fragmentation of the $m / z-254$ ion gave the most abundant product ion of $m / z$ 195, which is originated from the loss of a neutral $\left(\mathrm{CH}_{3}\right)_{3} \mathrm{~N}$ molecule from the GirT-derivatized 5-formyluracil (Figure 2b) ${ }^{11}$.

We next examined the yield for the conjugation reaction by varying the solution $\mathrm{pH}$, the molar ratio of the derivatizing reagent over FodU, and the reaction time. Because it was established that the rate for the formation of the hydrazone derivative is greatly enhanced under acidic conditions 15 , various amounts of acetic acid were often added to the reaction mixtures of Girard reagent with oxosteroids or oligosaccharides $10,11,16$. We also evaluated the efficiency for the conjugation reaction in phosphate buffer or in $10 \%$ acetic acid. Our results showed that, after a 12-h incubation at a GirT-to-FodU ratio of 10, the amount of hydrazone generated in phosphate buffer was only $35 \%$ of that produced in the corresponding reaction with the presence of $10 \%$ acetic acid, validating that the acid accelerates the formation of the GirT hydrazone. Nevertheless, with the GirT in large excess (e.g., GirT/FodU = 100:1) and with a reaction time of $12 \mathrm{~h}$, the amount of GirT hydrazone formed in phosphate buffer was comparable to that generated in $10 \%$ acetic acid solution, suggesting that, with the GirT in large excess, reaction under neutral $\mathrm{pH}$ can also allow for the efficient formation of the hydrazone derivative. Given that FodU and the resulting hydrazone are stable in $10 \%$ acetic acid solution, we added $10 \%$ acetic acid to the reaction mixtures for all the following derivatization reactions to achieve a high reaction rate.

We next investigated the yields for the formation of the conjugate at different molar ratios of GirT over FodU (from 2:1 to 2000:1, see Experimental Section). It turned out that the derivatization was efficient when the ratio reached 30:1, where 97.5\% of the starting FodU was consumed and the yield for the formation of the resulting hydrazone was about $98 \%$ of the highest yield from reactions where the GirT/FodU ratios were 100:1, 300:1, 1000:1 and 2000:1 (Figure 3a). In this respect, the percentage was calculated from the peak areas observed in the HPLC traces, which were recorded with UV detection at $281 \mathrm{~nm}$.

Our results also revealed that the conjugation between GirT and FodU occurred very rapidly. At a GirT-to-FodU ratio of 10, significant amount of GirT hydrazone was formed within 10 $\mathrm{min}$. The amount of the conjugate increases slightly as the reaction time increased from $10 \mathrm{~min}$ to $4 \mathrm{~h}$, and little increase was observed after $4 \mathrm{~h}$ (Figure $3 \mathrm{~b}$ ). While the GirT was in large excess (GirT:FodU = 100:1 and 1000:1), the formation of GirT hydrazone followed a similar trend (data not shown).

\section{Enhancement of ion signal upon derivatization}

FodU has a strong tendency to associate with $\mathrm{Na}^{+}$ion to give the $[\mathrm{M}+\mathrm{Na}]^{+}$ion (of $\left.m / z 279\right)$ in positive-ion ESI-MS. As a result, the $[\mathrm{M}+\mathrm{Na}]^{+}$ion dominated the positive-ion spectrum when $100 \mathrm{fmol}$ of FodU was injected for LC-MS analysis. In contrast, the $[\mathrm{M}+\mathrm{Na}]^{+}$and $[\mathrm{M}$ $+\mathrm{H}]^{+}$ions were produced in similar abundance when 2 pmol of FodU was injected. In addition, the product-ion spectrum of the electrospray-produced $[\mathrm{M}+\mathrm{Na}]^{+}$ion of FodU gave a single fragment ion of $m / z 163$, which is attributed to the loss of a 2-deoxyribose moiety (Figure S2). We failed to observe any product ion resulting from the further fragmentation of the ion of $\mathrm{m} /$ $z 163$ (data not shown). We reason that the failure might be attributed to the predominant formation of $\mathrm{Na}^{+}$ion, which could not be detected owing to the low $\mathrm{m} / \mathrm{z}$ limit of the linear ion trap. Consistent with this view, we found that the collisional activation of the $[\mathrm{M}+\mathrm{Cs}]^{+}$ion 
of 5-formyluracil ( $\mathrm{m} / \mathrm{z}$ 273) led to the predominant formation of the $\mathrm{Cs}^{+}$ion $(\mathrm{m} / \mathrm{z}$ 133) (Figure S3).

We then assessed the detection limit of FodU on our LC-MS/MS system where we monitored the fragmentation of the $[\mathrm{M}+\mathrm{Na}]^{+}$ion of FodU. It turned out that, at a signal-to-noise ratio $(\mathrm{S} / \mathrm{N})$ of 3, the LC-MS/MS detection limit of FodU on the LTQ linear-ion-trap mass spectrometer was $60 \mathrm{fmol}$. In this regard, it is worth mentioning that a detection limit of 30 fmol of FodU at an S/N of 3 was observed on a triple-quadrupole mass spectrometer operated in multiple-reaction monitoring (MRM) mode, where the $\mathrm{m} / \mathrm{z} 255 \rightarrow 212$ transition was monitored in the negative-ion mode ${ }^{7}$. We also attempted to detect FodU by LC-MS/MS in the negative-ion mode; the sensitivity, however, was at least 10 times poorer than that of the positive-ion mode where the fragmentation of the $[\mathrm{M}+\mathrm{Na}]^{+}$ion was monitored ${ }^{8}$.

It is worth noting that the detection limit of FodU in the digested DNA sample was much poorer than that of the pure standard. In this context, we spiked $10 \mu \mathrm{g}$ of digested DNA with 3-pmol FodU and injected the sample for LC-MS/MS analysis, where the fragmentation of the $[\mathrm{M}+$ $\mathrm{Na}]^{+}$ion of FodU was recorded. Surprisingly, no obvious peak for FodU was observed in the SIC for monitoring the $m / z 279 \rightarrow 163$ transition, suggesting that direct LC-MS/MS analysis in the positive-ion mode may not be an appropriate method for the detection and quantification of low level of FodU in the digested DNA samples.

We next assessed the limit of detection for FodU based on the LC-MS/MS and LC-MS ${ }^{3}$ analyses of the derivatization mixture of FodU. The LC-MS/MS detection limit based on monitoring the $m / z, 370 \rightarrow 254$ transition was determined to be $4.3 \mathrm{fmol}$ at an $\mathrm{S} / \mathrm{N}$ of 3 , which was approximately 14 times better than the underivatized FodU analyzed on the same instrument under similar conditions (vide supra). We also evaluated the limit of detection for FodU by monitoring the $m / 2370 \rightarrow 254 \rightarrow 195$ transition and we found that the LC-MS ${ }^{3}$ method had a slightly better detection limit, that is, $3.3 \mathrm{fmol}$ at an $\mathrm{S} / \mathrm{N}$ of 3, than that obtained by LCMS/MS. This level of sensitivity can allow one to detect FodU from $1 \mu \mathrm{g}$ of DNA if FodU is formed at a frequency of 1 lesion per $10^{6}$ normal nucleosides. In this context, it is worth mentioning that the sensitivity should be further improved if the derivative is analyzed by LCMS/MS on a triple quadrupole instrument operated in the MRM mode. It should also be added that the detection limit of the derivatized FodU in the digested DNA samples might be somewhat poorer than that for the pure standard due to the suppression of the ionization by the presence of co-eluted unmodified nucleosides or other species in the biological matrix.

Nevertheless, we were able to detect readily $50 \mathrm{fmol}$ of FodU when it was spiked with $10 \mathrm{nmol}$ of each of the four unmodified 2'-deoxynucleosides (data not shown).

When compared with LC-MS/MS, LC-MS ${ }^{3}$ provided better specificity in identifying and quantifying FodU, especially for FodU present in the digestion mixtures of DNA. In this respect, we analyzed a sample where $10 \mu \mathrm{g}$ of hydrolyzed DNA, which was obtained from Hela S3 cells that were treated with 66 Gy of $\gamma$-rays, was doped with 3 pmol of isotope-labeled FodU. Two fragmentation events, i.e., MS/MS and $\mathrm{MS}^{3}$, recording the fragmentation of the ion of $\mathrm{m} / \mathrm{z} 373$ and the further fragmentation of the ion of $\mathrm{m} / \mathrm{z} 256$ observed in MS/MS, respectively, were alternatively monitored. It turned out that only one peak eluting at the expected retention time (i.e., $14.7 \mathrm{~min}$ ) for the derivatized FodU was present in the SIC for the $\mathrm{m} / \mathrm{z} 373 \rightarrow 256 \rightarrow 197$ transition, whereas two peaks (at 12.2 and $14.7 \mathrm{~min}$ ) were found in the SIC for the $m / z, 373 \rightarrow 256$ transition (Figure S4). The 12.2-min fraction is due to the presence of impurity in the digestion mixture. Considering the high specificity of LC-MS ${ }^{3}$ as well as its superior detection limit, we chose to employ LC-MS ${ }^{3}$, together with derivatization with GirT, to quantify the amount of FodU formed in cellular DNA. 


\section{LC-MS quantifications of derivatized FodU in cellular DNA}

With the establishment of a sensitive detection method and the availability of the isotopelabeled FodU, we next employed the method to quantify the yield for the formation of this oxidative lesion in human Hela-S3 cells upon exposure to $\gamma$-rays. To this end, we first constructed a calibration curve, where we mixed the unlabeled and labeled FodU at a series of molar ratios, treated the samples with GirT, and analyzed the resulting reaction mixtures with LC-MS. It turned out that the measured ratios of the unlabeled and labeled hydrazones were almost identical to the actual ratios between the unlabeled and labeled FodU that we added (Figure S5). Therefore, the derivatization of FodU by GirT could be used for the reliable quantification of FodU.

We next exposed the Hela-S3 cells to a series of doses of $\gamma$-rays, isolated the cellular DNA, and digested the DNA by a combination of four enzymes (see Experimental Section). We then added the isotope-labeled FodU to the digestion mixture, removed most unmodified nucleosides by HPLC, derivatized the FodU-containing fractions with GirT, and analyzed the derivatization mixture by $\mathrm{LC}_{\mathrm{MS}}{ }^{3}$.

The SIC for monitoring the $m / z, 370 \rightarrow 254 \rightarrow 195$ transition for the unlabeled GirT hydrazone showed a fraction eluting at the same time as the labeled internal standard (Figure 4). The product-ion spectra shown in the insets further supported the presence of FodU in the enzymatic digestion mixture of cellular DNA isolated from $\gamma$-irradiated cells. Furthermore, the quantification results indicated that the yield for the formation of FodU was proportional to the dose of $\gamma$-rays that the cells received, with a lesion formation rate of 0.011 lesion $/ 10^{6}$ bases per Gray in Hela-S3 cells (Figure 5).

This result is in reasonable agreement with a formation rate of 0.022 lesion $/ 10^{6}$ bases per Gray reported by Pouget et al. ${ }^{17}$ in the viewpoint that different cell lines were used and the $\gamma$-ray exposures were carried out at different dose rates. In the former respect, these authors used THP-1 cells, whereas Hela S3 cells were employed in our study. In addition, Pouget et al. ${ }^{17}$ delivered the $\gamma$-rays with a ${ }^{60} \mathrm{Co}$ source at a dose rate at $18 \mathrm{~Gy} / \mathrm{min}$, whereas the dose rate delivered by our ${ }^{137} \mathrm{Cs}$ irradiator was $2.8 \mathrm{~Gy} / \mathrm{min}$. The lower dose rate required longer exposure time for the irradiation at the same dose, which may lead to more extensive repair of the lesion under study. Thus, we observed somewhat lower levels of 5-FodU.

\section{Conclusions}

The key advantage of our method is that derivatization with GirT dramatically enhanced the sensitivity for the detection and quantification of FodU in cellular DNA samples, making it feasible to detect FodU from a small amount of DNA sample isolated from cultured human cells or tissues. With the availability of the isotope-labeled internal standard, we were able to quantify FodU in cellular DNA with significantly improved sensitivity. On the grounds that FodU is one of the major oxidative products of thymidine induced by ROS, the highly sensitive method reported in this paper may facilitate the possible application of FodU as a biomarker for monitoring the exposures to genotoxic agents that cause oxidative DNA damage. In addition, we envision that the method can be applied for the sensitive detection of other oxidative DNA lesions including the abasic site and 5-formyl-2'-deoxycytidine. Moreover, out study further broadened the application of Girard reagent for the highly sensitive quantification of ketone/aldehyde-containing compounds.

\section{Supplementary Material}

Refer to Web version on PubMed Central for supplementary material. 


\section{Acknowledgement}

The authors thank the National Institutes of Health for supporting this research (R01 CA96906), Dr. Huachuan Cao for synthesizing the isotope-labeled FodU, and the National Cell Culture Center for culturing human Hela-S3 cells.

\section{References}

(1). Lindahl T. NATO ASI Ser., Ser. A 1999;302:251-257.

(2). Finkel T, Holbrook NJ. Nature 2000;408:239-247. [PubMed: 11089981]

(3). Marnett LJ. Carcinogenesis 2000;21:361-370. [PubMed: 10688856]

(4). Zhang QM, Sugiyama H, Miyabe I, Matsuda S, Saito I, Yonei S. Nucleic Acids Res 1997;25:3969_ 3973. [PubMed: 9321644]

(5). Masaoka A, Terato H, Kobayashi M, Ohyama Y, Ide H. J. Biol. Chem 2001;276:16501-16510. [PubMed: 11278425]

(6). Kamiya H, Murata-Kamiya N, Karino N, Ueno Y, Matsuda A, Kasai H. Mutat. Res 2002;513:213222. [PubMed: 11719107]

(7). Frelon S, Douki T, Ravanat JL, Pouget JP, Tornabene C, Cadet J. Chem. Res. Toxicol 2000;13:10021010. [PubMed: 11080049]

(8). Hong H, Cao H, Wang Y, Wang Y. Chem. Res. Toxicol 2006;19:614-621. [PubMed: 16696563]

(9). Higashi T, Shimada K. Anal. Bioanal. Chem 2004;378:875-882. [PubMed: 14598004]

(10). Gouw JW, Burgers PC, Trikoupis MA, Terlouw JK. Rapid Commun. Mass Spectrom 2002;16:905912. [PubMed: 11968119]

(11). Shackleton CH, Chuang H, Kim J, de la Torre X, Segura J. Steroids 1997;62:523-529. [PubMed: 9253791]

(12). Mirzaei H, Regnier F. Anal. Chem 2006;78:770-778. [PubMed: 16448050]

(13). Lai CC, Tsai CH, Tsai FJ, Lee CC, Lin WD. Rapid Commun. Mass Spectrom 2001;15:2145-2151. [PubMed: 11746879]

(14). Wang Y, Hornshaw M, Alvelius G, Bodin K, Liu S, Sjovall J, Griffiths WJ. Anal. Chem 2006;78:164-173. [PubMed: 16383324]

(15). Wheeler O, Rosado-Lojo O. Tetrahedron 1962;18:477-482.

(16). Griffiths WJ, Liu S, Alvelius G, Sjovall J. Rapid Commun. Mass Spectrom 2003;17:924-935. [PubMed: 12717765]

(17). Pouget JP, Frelon S, Ravanat JL, Testard I, Odin F, Cadet J. Radiat. Res 2002;157:589-595. [PubMed: 11966325] 
<smiles>O=Cc1cn(C2CC(O)C(CO)O2)c(=O)[nH]c1=O</smiles><smiles>C[N+](C)(C)CC(=O)NN</smiles><smiles>C[N+](C)(C)CC(=O)N/N=C/c1cn(C2CC(O)C(CO)O2)c(=O)[nH]c1=O</smiles>

Figure 1.

The reaction of FodU with GirT. 

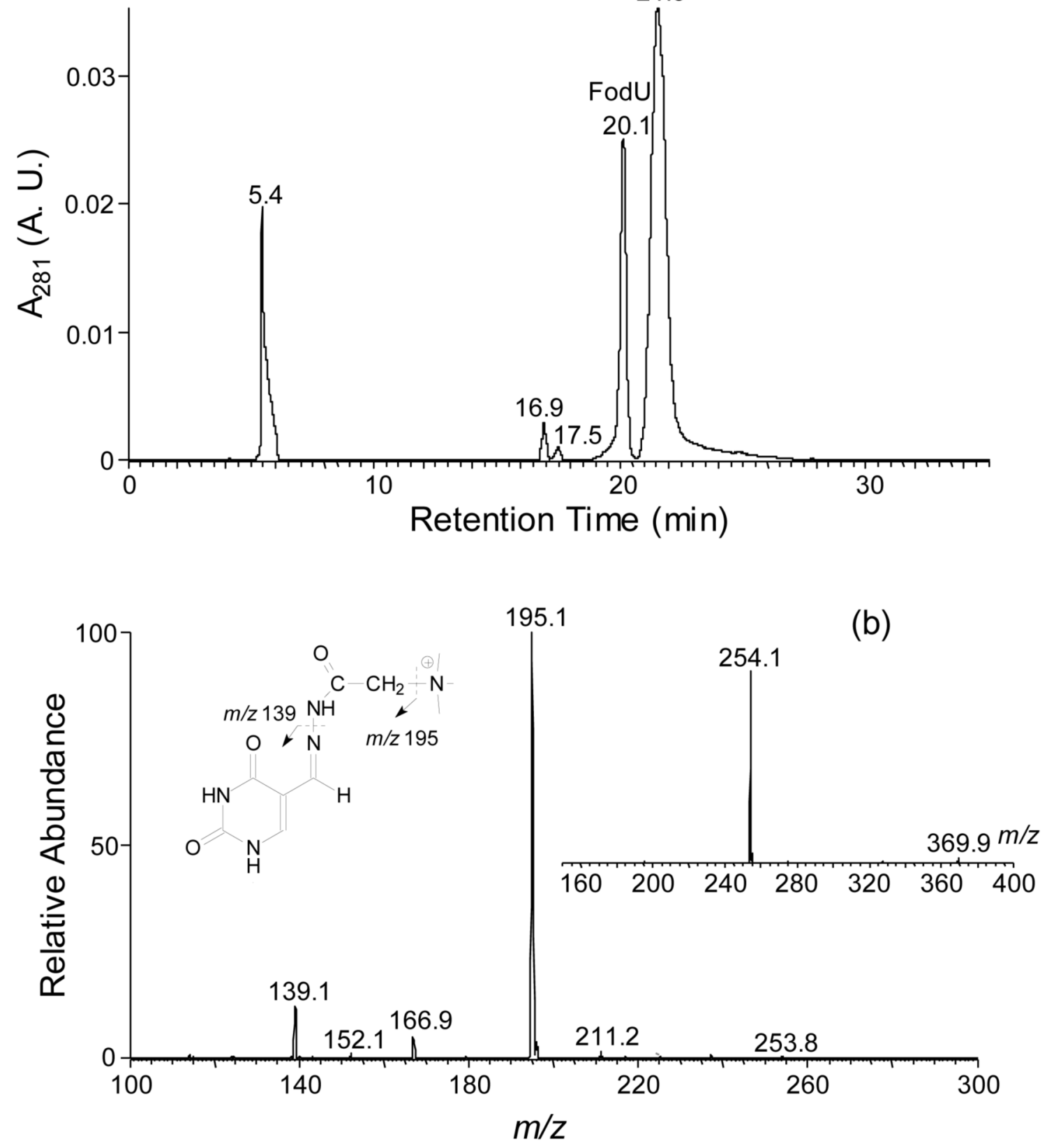

Figure 2.

(a) The HPLC trace for the separation of the reaction mixture where FodU was treated with GirT at a molar ratio of 1:2 at room temperature for $12 \mathrm{~h}$. (b) ESI-MS ${ }^{3}$ of the ion of $m / z 254$ observed in MS/MS; shown in the inset are the MS/MS of the $[\mathrm{M}+\mathrm{H}]^{+}$ion of the hydrazone conjugate and the cleavage reactions for the formation of major fragment ions found in $\mathrm{MS}^{3}$. 

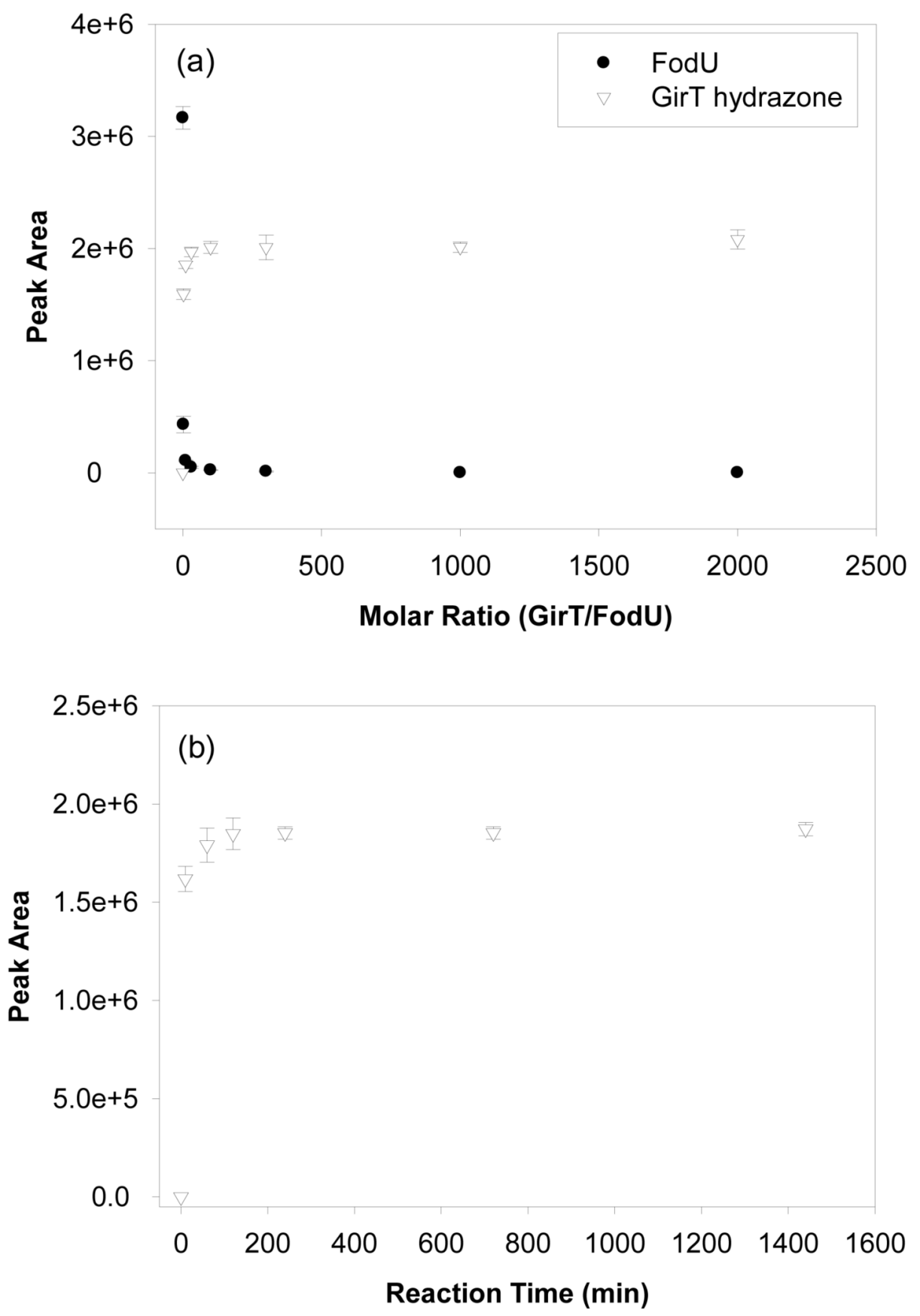

Figure 3.

(a) Optimization of the molar ratios of GirT over FodU for the derivatization. GirT was mixed with FodU at molar ratios of 2:1, 10:1, 30:1, 100:1, 300:1, 1000:1 and 2000:1 in the presence of $10 \%$ acetic acid, and the reactions were continued at room temperature for $12 \mathrm{~h}$. Plotted are the HPLC peak areas for FodU and the hydrazone conjugate. (b) Optimization of the derivatization time. GirT was mixed with FodU at a molar ratio of 10:1 in the presence of 10\% acetic acid, and the reactions were carried out at room temperature for time periods of $10 \mathrm{~min}$ and 1, 2, 4, 12 and $24 \mathrm{~h}$, respectively. Plotted are the HPLC peak areas for the hydrazone conjugate (the fractions were monitored by UV detection at $281 \mathrm{~nm}$ ). The values represent the means \pm SD from three independent measurements. 


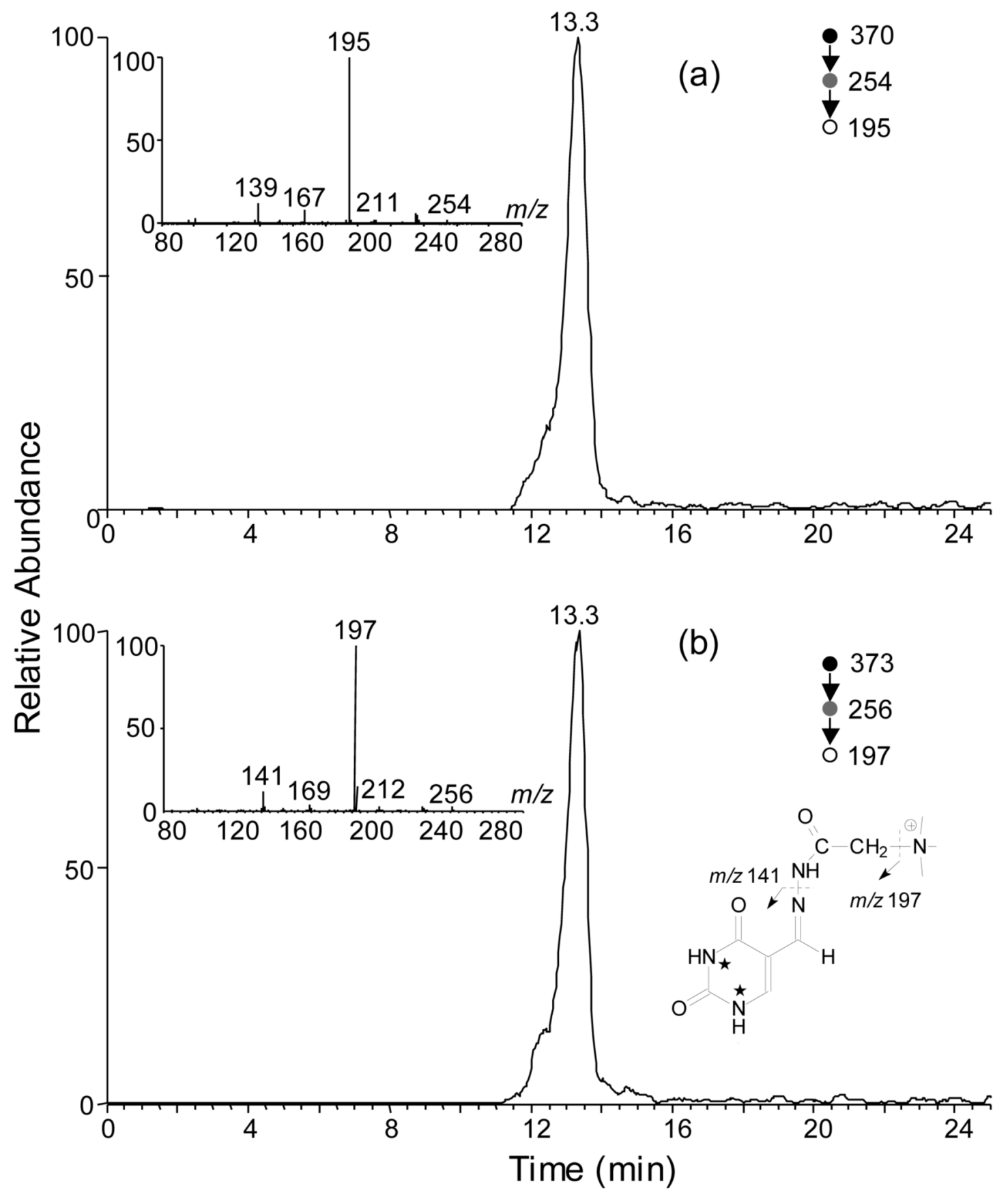

Figure 4.

SICs for the monitoring of the $m / z, 370 \rightarrow 254 \rightarrow 195$ [a, for unlabeled FodU-GirT hydrazone] and $m / z, 373 \rightarrow 256 \rightarrow 197$ [b, for isotopically labeled FodU-GirT hydrazone] transitions of the digestion mixtures of the cellular DNA samples, which were extracted from Hela-S3 cells treated with $110 \mathrm{~Gy}$ of $\gamma$-rays. Shown in the insets are the $\mathrm{MS}^{3}$ of the ions of $\mathrm{m} / z 254$ and 256 for the unlabeled and labeled hydrazones, respectively. The "*" in the structure shown in the inset of panel $b$ designates that the nitrogen atoms are replaced with an " $15 \mathrm{~N}$ ". 


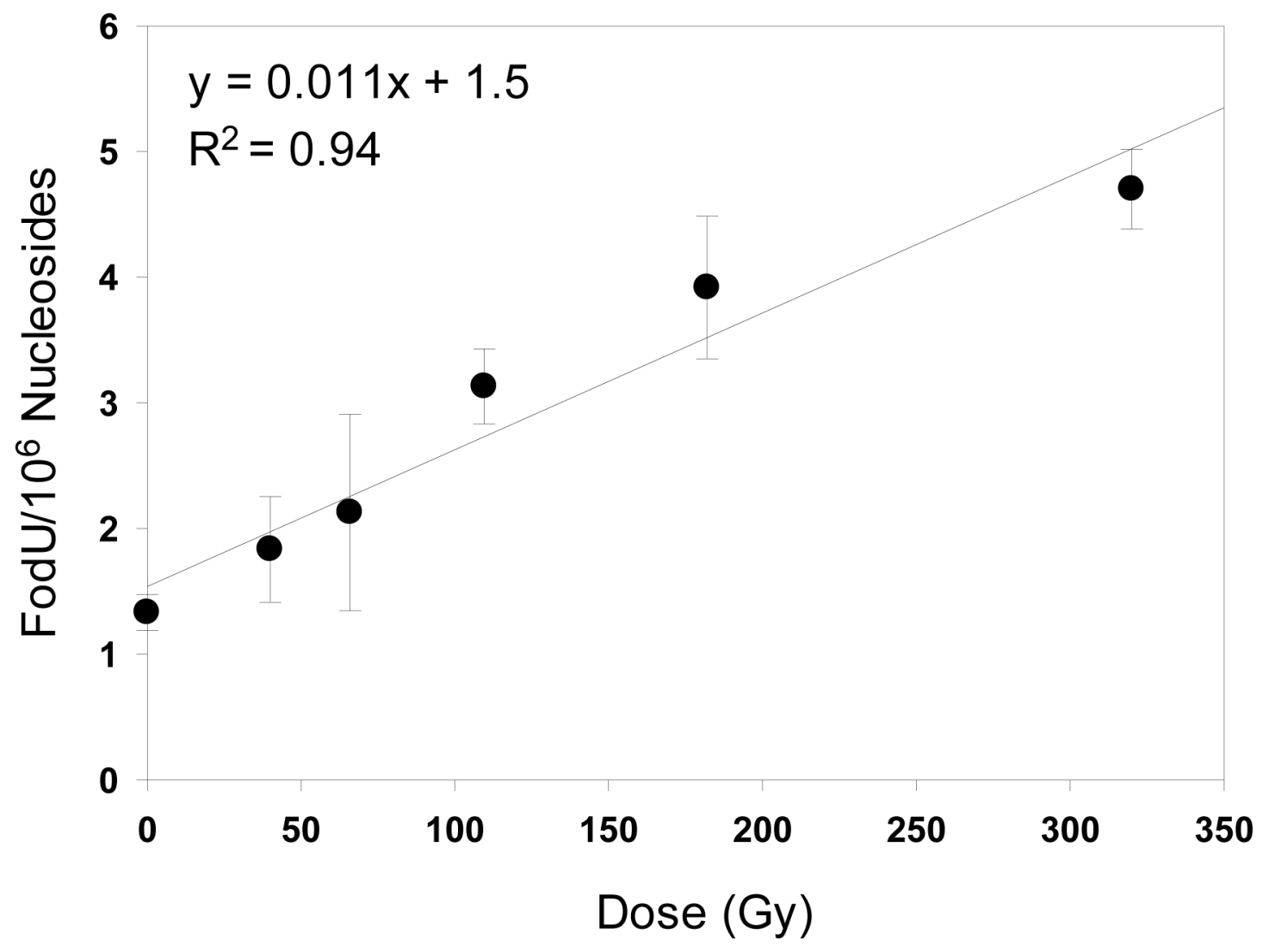

Figure 5.

Dose-dependent formation of FodU in Hela-S3 cells upon exposure to $\gamma$-rays. The values represent the means \pm SD from three independent measurements. 